\title{
COMPUTATION OF THE DEMAGNETIZING POTENTIAL IN MICROMAGNETICS USING A COUPLED FINITE AND INFINITE ELEMENTS METHOD
}

\author{
François Alouges ${ }^{1}$
}

\begin{abstract}
This paper is devoted to the practical computation of the magnetic potential induced by a distribution of magnetization in the theory of micromagnetics. The problem turns out to be a coupling of an interior and an exterior problem. The aim of this work is to describe a complete method that mixes the approaches of Ying [12] and Goldstein [6] which consists in constructing a mesh for the exterior domain composed of homothetic layers. It has the advantage of being well suited for catching the decay of the solution at infinity and giving a rigidity matrix that can be very efficiently stored. All aspects are described here, from the practical construction of the mesh, the storage of the matrix, the error estimation of the method, the boundary conditions and a simple preconditionning technique. At the end of the paper, a typical computation of a uniformly magnetized ball is done and compared to the analytic solution. This method gives a natural alternatives to boundary elements methods for 3D computations.
\end{abstract}

Mathematics Subject Classification. 65G99, 65Y20, 75Z05, 78A30, 78M10.

Received May 3, 2000. Revised May, 2001.

\section{INTRODUCTION AND PRELIMINARY OBSERVATIONS}

In the classical theory of micromagnetics [2], the magnetization $\vec{u}$ present in a ferromagnetic body (a closed bounded regular domain of $\mathbb{R}^{3}$ called $\Omega$ in the sequel) generates the so-called demagnetizing field $H=\nabla \phi$, where $\phi$ is obtained, up to a physical factor, by the Helmholtz decomposition of $\vec{u}$ (also called Hodge-De Rham decomposition when working with differentiable forms)

$$
\vec{u}=\nabla \phi+\operatorname{curl}(\psi)
$$

It is important to notice furthermore that in (1), the decomposition is made in the sense of distributions on all $\mathbb{R}^{3}$ extending $\vec{u}$ by 0 outside $\Omega$. Moreover, the decomposition (1) is unique provided $\phi$ and $\psi$ decrease to 0 at infinity and $\operatorname{div} \psi=0$ for instance.

Keywords and phrases: Micromagnetics, finite element method, preconditionning, exterior problems.

1 Laboratoire de Mathématiques, Université d'Orsay, 91405 Orsay Cedex, France; e-mail: Francois.Alouges@math.u-psud.fr 
Denoting by $\omega$ the complementary of $\Omega$, and $\Gamma$ the boundary of $\Omega$, it can be seen by taking the divergence of (1) that $\phi$ is solution to the elliptic problem

$$
\left\{\begin{array}{l}
\Delta \phi=\operatorname{div}(\vec{u}) \text { in } \Omega, \\
\Delta \phi=0 \text { in } \omega, \\
{\left[\frac{\partial \phi}{\partial n}\right]=\vec{u} \cdot \vec{n} \text { across } \Gamma .}
\end{array}\right.
$$

Here, we use the symbol $[f]$ to denote the jump $f_{\text {int }}-f_{\text {ext }}$ of a quantity $f$ across $\Gamma$ and $\vec{n}$ is the unit normal pointing outside $\Omega$.

From (2), it is possible to express the scalar potential $\phi$ in terms of the fundamental solution to the Laplace equation in $\mathbb{R}^{3}$

Namely, we have

$$
G(x)=-\frac{1}{4 \pi|x|} .
$$

$$
\phi(x)=-\frac{1}{4 \pi} \int_{\Omega} \frac{\operatorname{div}(\vec{u}(y))}{|x-y|} \mathrm{d} y+\frac{1}{4 \pi} \int_{\Gamma} \frac{\vec{u}(y) \cdot \vec{n}(y)}{|x-y|} \mathrm{d} \sigma_{y},
$$

which after integrating by parts yields to

$$
\phi(x)=-\frac{1}{4 \pi} \int_{\Omega} \frac{\vec{u}(y) \cdot(x-y)}{|x-y|^{3}} \mathrm{~d} y .
$$

It is not difficult to see that the previous formula has a meaning at least if $\vec{u}$ belongs to $L^{\infty}(\Omega)$, which is typically the case in micromagnetic computations where the magnetization is constrained to be of constant magnitude inside the material [2].

Unfortunately, equation (4) is not of practical use for the numerical computation of $\phi$. Indeed, except in special cases (on Cartesian grids for instance it is recommended to use a fast Fourier transform), the fact that it involves a convolution will usually give rise to a dense matrix after discretization. The size of this dense matrix being proportional to the square of the number of discretization points used in $\Omega$, this forbids fine meshes on $\Omega$ and hence a correct description of $\vec{u}$.

In order to avoid the use of this formula, we prefer to write the variational formulation of (2). Following the standard theory (see e.g. [4]), we call

$$
H^{1, *}=\left\{\phi \text { such that } \nabla \phi \in L^{2}\left(\mathbb{R}^{3}\right), \text { and } \frac{\phi(x)}{\left(1+|x|^{2}\right)^{\frac{1}{2}}} \in L^{2}\left(\mathbb{R}^{3}\right)\right\} .
$$

$H^{1, *}$ is a Hilbert space when equipped either with the norm

$$
\|\phi\|_{1, *}^{2}=\int_{\mathbb{R}^{3}}|\nabla \phi(x)|^{2} \mathrm{~d} x+\int_{\mathbb{R}^{3}} \frac{\phi^{2}(x)}{1+|x|^{2}} \mathrm{~d} x,
$$

or with

$$
|\phi|_{1, *}^{2}=\int_{\mathbb{R}^{3}}|\nabla \phi(x)|^{2} \mathrm{~d} x
$$

The variational formulation of the problem reads

$$
\text { Find } \phi \text { in } H^{1, *} \text { such that } \int_{\mathbb{R}^{3}} \nabla \phi \cdot \nabla \psi \mathrm{d} x=\int_{\Omega} \vec{u} \cdot \nabla \psi \mathrm{d} x \text {, for all } \psi \in H^{1, *} \text {. }
$$


Using this formulation, it is not a difficult problem to write a finite elements discretization. The only remaining difficulty is that we need to consider a mesh of all $\mathbb{R}^{3}$ in order to compute the rigidity matrix of the problem. This question has been addressed by some authors and several techniques have been used to circumvent the problem. The simplest one is to consider a box $B$ containing $\Omega$ and approximate the original problem by

$$
\text { Find } \phi \text { in } H_{0}^{1}(B) \text { such that } \int_{B} \nabla \phi \cdot \nabla \psi \mathrm{d} x=\int_{\Omega} \vec{u} \cdot \nabla \psi \mathrm{d} x \text {, for all } \psi \in H_{0}^{1}(B) .
$$

Here $H_{0}^{1}(B)$ is the classical Sobolev space of functions in $H^{1}(B)$ vanishing on $\partial B$.

In this case the difficulty is to tune the size of $B$. Clearly $B$ must be taken rather large (remember that $\phi$ decays only algebraically by (4)). Moreover, if the meshes of $B \backslash \Omega$ and $\Omega$ are equally fine, the outside mesh may contain a lot more elements than the inside mesh. Hence, the numerical algorithm will spend a lot more time and memory to take into account the exterior problem than to solve the interior one.

To correct the preceding drawbacks, some authors send the boundary of $B$ to infinity by an inversion. This is more precise, but still requires a lot of elements outside $\Omega[3,9,10]$.

A totally different approach consists in using the theory of integral equations. Roughly speaking, remarking that $\phi$ is harmonic outside $\Omega$ and using the Green kernel of the Laplace operator like in formula (3) it is possible to solve a coupled problem on $\Omega$ and $\Gamma$ which involves a nonlocal operator on $\Gamma$ [4]. This kind of methods are called boundary elements methods and face a major difficulty: after discretization, the non-local operator leads to a dense matrix which therefore requires a storage proportional to the square of the number of unknowns on $\Gamma$. Hence, it is very difficult to use them in 3 -D situations if the mesh of $\Gamma$ is fine.

At last, it has been remarked that it is possible to use a mesh of $\omega$ such that the mesh size increases at infinity [6]. Indeed, if the increase is not too fast, it will be compensated by the decay of the solution and its derivatives, and it is possible to derive a good error estimation.

Quite surprisingly, such increase rates are obtained by the construction of Ying [12] in his "infinite elements method" 2 (see also [11]) who builds the mesh as a superposition of homothetic layers of tetraedra. On the other hand both Silvester et al. and Ying used this kind of construction in order to get a good discretization of the Dirichlet to Neumann operator on $\Gamma$ (thus falling back to the problem of storing the dense matrix already mentionned).

The purpose of this paper is to provide a complete method that can be used for 3-D computations. Namely, we give an automatic procedure to build the exterior mesh from the boundary mesh of $\Omega$. Such a graded mesh falls directly into the theories of Goldstein (another proof of the error estimate is also given) and Ying. The fact that the exterior mesh is composed of homothetic layers is crucial and leads to nice storage properties of the outside matrix. Subsequently, we also give a way to simplify the fomulation by making a change of variables of the outside unknowns. As the numerical experiments show, this modification acts like a preconditionner and speeds up the algorithm. Eventually, the numerical experiments provided in the paper make a comparison with respect to the kind of boundary conditions used, the number of layers, and the number of iterations used to solve the system.

The paper is divided as follows. The principle of discretization is recalled in Section 2. For the convenience of the reader and because the methodology developed here is slightly different than the one of Goldstein, we make the error estimation on the mesh consisting of an infinite number of layers in Section 3. Then, the truncation of the domain (which leads to a finite dimensional system) is explained in Section 4, where we also discuss the boundary conditions to apply on the outer boundary. We also discuss the automatic construction of the exterior mesh and a simple but efficient preconditionning technique. In Section 5, the example consisting of a uniformly magnetized ball (where the solution is known explicitly) is presented.

At last, the reader might be surprised of the simplicity of the problem considered (classical magnetostatics) and might wonder why the magnetization is taken as a given data since it is usually unknown (and depends

\footnotetext{
${ }^{2} \mathrm{Be}$ careful, this method has nothing to do with the usual infinite element method where the approximation functions have an infinite support. Here, every basis function has a compact support but there are infinitely many of them.
} 
often nonlinearly on material constants and other quantities like the field). The reason for that is that this simple computation is often a brick which goes usually inside an iterative loop (think to a Newton's method for example). In a forthcoming work, we will apply the method described here to compute the magnetization field obeying the theory of micromagnetics developed in [2].

\section{INFINITE ELEMENTS METHOD AND THEORETICAL FRAMEWORK}

As described in [12], when we use the infinite elements method to solve an exterior problem, we first build a mesh of $\omega$ composed of homothetic layers. The principle is simple but requires $\Gamma$ to be a convex polyedron ${ }^{3}$. We consider a center $O$ in $\Omega$ and a constant $\xi>1$. Then, for all integer $k$, we call $\Gamma_{k}=\xi^{k} \Gamma$, the convex polyedron homothetic to $\Gamma$ with constant of proportionality $\xi^{k}$ and center $O$.

The domain $C_{k}$ delimited by two consecutive polyedra $\Gamma_{k}$ and $\Gamma_{k+1}$ is called a "layer", and eventually, the layers are decomposed into tetraedra in such a manner that the partition is identical for each layer. A simple example of multi-layers mesh is drawn in Figure 1.

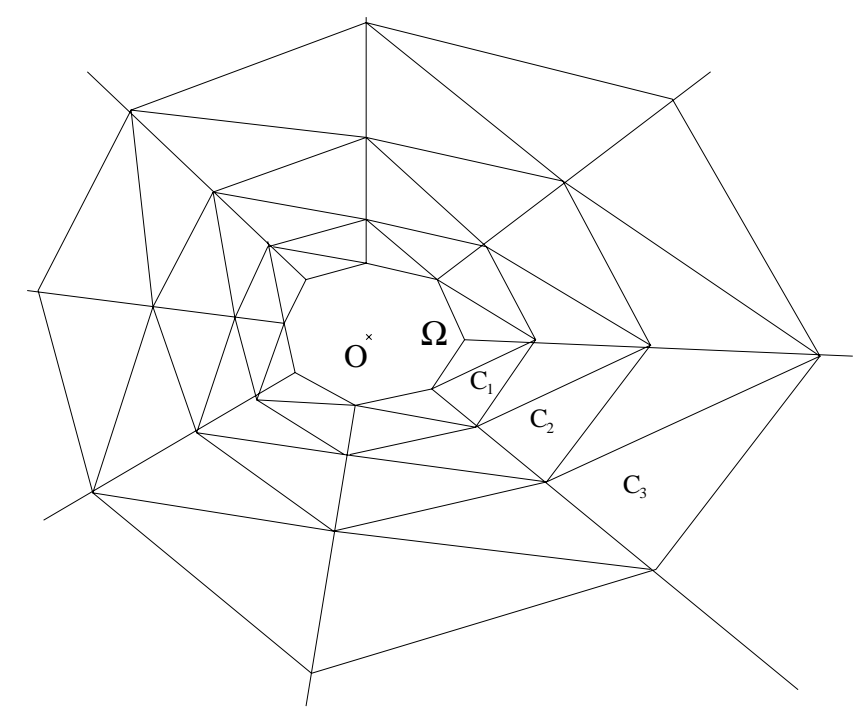

FiguRE 1. An example of multi-layers mesh outside a domain $\Omega$.

We thus obtain a conformal finite element mesh of $\omega$. It is very important to already notice that at infinity, the tetraedra of the triangulation become larger and larger.

In [12] or [11], the authors iteratively builds from the rigidity matrix of one layer, the rigidity matrix of 2,4 , $\cdots, 2^{k} \cdots$ consecutive layers. They then prove that this sequence of matrices quickly converges to the rigidity matrix of the exterior infinite mesh (this is exactly the discretized Dirichlet-Neumann operator ${ }^{4}$ ).

We take here a different approach. We keep the multi-layers mesh of $\omega$, and remark that it is graded in the sense used in [6]. Then we couple it to an interior mesh of $\Omega$ and solve our problem on this mesh of $\mathbb{R}^{3}$. The size of the elements far from $\Gamma$ is compensated by the magnitude of the derivative of the solution $\phi$ which leads to an error estimation independent of the number of layers considered and as precise as if the meshes of $\Omega$ and $\omega$ were equally fine. This has been already proved by Goldstein [6] but we provide another slightly different proof in the next section.

\footnotetext{
${ }^{3}$ This is not a crucial obstruction since it is possible to rewrite the problem on $\Omega^{\prime}$ the convexified of $\Omega$.

${ }^{4}$ The advantage of this method being that the matrices recursively constructed are all symmetrical positive definite (refer to [12] for details). However, if the method is theoretically nice, numerical instabilities may occur when computing the sequence.
} 
As usual, we introduce the classical following notations. If $K$ is a tetraedron, we call $\rho_{K}$ the diameter of the largest sphere included in $K$, and $h_{K}$ the diameter of $K$. By extension, if $\mathcal{T}$ is a union of tetraedra (a triangulation for instance), we denote by $h_{\mathcal{T}}$ its aspect-ratio defined by

$$
h_{\mathcal{T}}=\sup _{K \in \mathcal{T}} h_{K}
$$

Let $\mathcal{T}_{k}$ a sequence of triangulations of $\Omega$. We call $\overline{\mathcal{T}_{k}}$ the triangulation ${ }^{5}$ of $\mathbb{R}^{3}$ obtained by gluing on $\Gamma$ the triangulation $\mathcal{T}_{k}$ and the multi-layers triangulation of $\omega$ described before.

We assume that this sequence is regular in the sense that there exists $C>0$ such that

$$
\forall k, \sup _{K \in \mathcal{T}_{k} \cup C_{1}} \frac{h_{K}}{\rho_{K}} \leq C,
$$

and moreover, such that $h_{\mathcal{T}_{k} \cup C_{1}} \longrightarrow 0$ when $k \longrightarrow \infty$.

We insist on the fact that it does not imply a dependence of $\xi$ on $h_{\mathcal{T}}$. We just need to have a triangulation of $C_{1}$ as "fine" as $\mathcal{T}$.

The space $H_{h}^{1, *}$ which is a discretized version of $H^{1, *}$ is simply the space of function that are linear on each element of $\overline{\mathcal{T}}$

$$
H_{h}^{1, *}=\left\{\phi \in H^{1, *} \text { such that }\left.\phi\right|_{K} \in P^{1}(K) \forall K \in \overline{\mathcal{T}}\right\} .
$$

One can remark that although $H_{h}^{1, *}$ is a discretized space, it is nonetheless of infinite dimension since the triangulation $\overline{\mathcal{T}}$ contains infinitely many tetraedra.

From Lax-Milgram theorem, it is easily seen that the problem

$$
\text { Find } \phi_{h} \in H_{h}^{1, *} \text { such that } \int_{\mathbb{R}^{3}} \nabla \phi_{h} \cdot \nabla \psi_{h} \mathrm{~d} x=\int_{\Omega} \vec{u} \cdot \nabla \psi_{h} \mathrm{~d} x \text { for all } \psi_{h} \in H_{h}^{1, *} \text {, }
$$

has a unique solution.

The remaining problem is to evaluate the error between the exact solution $\phi$ and the approximate $\phi_{h}$. In the following section, we prove that the classical error estimation (see [4] for example)

$$
\left|\phi-\phi_{h}\right|_{1, *} \leq C h_{\mathcal{T} \cup C_{1}},
$$

remains valid although the size of the elements increases at infinity, provided the vector-field $\vec{u}$ is chosen to belong to the Sobolev space ${ }^{6} H^{1}\left(\Omega, \mathbb{R}^{3}\right)$.

\section{ERror estimate}

In this section, we prove the error estimate. Although the result can typically be found in [6], we provide it to the reader in a slightly different way. Indeed here, we get the error estimation on the infinite mesh and truncate it afterwards.

The classical method consists in bounding the error by an interpolation error. More precisely using classical techniques (and noticing that the bilinear form $a(\phi, \psi)=\int_{\mathbb{R}^{3}} \nabla \phi \cdot \nabla \psi \mathrm{d} x$ is coercive on $H^{1, *}$ ) one easily gets

$$
\left|\phi-\phi_{h}\right|_{1, *} \leq C \inf _{\psi_{h} \in H_{h}^{1, *}}\left|\phi-\psi_{h}\right|_{1, *}
$$

\footnotetext{
${ }^{5}$ When no confusion is possible we simply note $\mathcal{T}$ instead of $\mathcal{T}_{k}, \overline{\mathcal{T}}$ instead of $\overline{\mathcal{T}}_{k}$.

${ }^{6}$ For micromagnetic calculations this is not a restriction [2].
} 
Now following the usual reasoning, we estimate the right hand side term using the second derivative of the exact solution $\phi$ to get

$$
\left|\phi-\phi_{h}\right|_{1, *}^{2} \leq C \sum_{K \in \overline{\mathcal{T}}}|\phi|_{H^{2}(K)}^{2} h_{K}^{2},
$$

where the semi-norm $|\phi|_{H^{2}(K)}$ is the $L^{2}(K)$ norm of the second derivative $D^{2} \phi$ of $\phi$.

Now, we need to express the fact that at infinity the $H^{2}$ semi-norm of $\phi$ decays and compensates the increase of $h_{K}$. This is precisely the object of the following lemmas and theorem.

Lemma 1 (Regularity). Assume $\vec{u}$ belongs to the space $H^{1}(\Omega)$. Then the potential $\phi$ solution to (5) belongs to the space $\dot{H}^{2}(\Omega) \times \dot{H}^{2}(\omega)=\left\{\phi \in H^{1, *}\left(\mathbb{R}^{3}\right)\right.$ such that $\left.D^{2} \phi\right|_{\Omega} \in L^{2}(\Omega)$ and $\left.\left.D^{2} \phi\right|_{\omega} \in L^{2}(\omega)\right\}$.

Proof. We decompose $\phi$ into two parts $\phi_{1}$ and $\phi_{2}$ as implicitly described in formula (3)

$$
\phi_{1}(x)=-\frac{1}{4 \pi} \int_{\Omega} \frac{\operatorname{div}(\vec{u}(y))}{|x-y|} \mathrm{d} y,
$$

and

$\phi_{1}$ is thus solution to

$$
\phi_{2}(x)=\frac{1}{4 \pi} \int_{\Gamma} \frac{\vec{u}(y) \cdot \vec{n}(y)}{|x-y|} \mathrm{d} \sigma_{y} .
$$

$$
\Delta \phi_{1}=\operatorname{div}(\vec{u}) \chi_{\Omega},
$$

in $\mathbb{R}^{3}$ and thus belongs to $\dot{H}^{2}\left(\mathbb{R}^{3}\right)=\left\{\phi \in H^{1, *}\right.$ such that $\left.D^{2} \phi \in L^{2}\left(\mathbb{R}^{3}\right)\right\}$ (since $\vec{u} \in L^{2}(\Omega)$ ).

Moreover since the operator:

$$
\Psi:\left.g \longrightarrow \int_{\Gamma} g(y) \frac{\partial}{\partial n_{y}}\left(\frac{1}{|x-y|}\right) d \sigma_{y}\right|_{\Gamma}
$$

maps $H^{s}(\Gamma)$ into $H^{s+1}(\Gamma)$ (see e.g. [4]), we deduce that the trace of $\phi_{2}$ on $\Gamma$ and hence the trace $\left.\phi\right|_{\Gamma}$ of $\phi$ on $\Gamma$ belongs to $H^{3 / 2}(\Gamma)$. A standard regularity result gives that $\phi$ belongs to $H^{2}(\Omega)$ (since $\Delta \phi=\operatorname{div}(\vec{u}) \in L^{2}(\Omega)$ and $\left.\left.\phi\right|_{\Gamma} \in H^{3 / 2}(\Gamma)\right)$.

For the outer part of $\phi$, we take $\bar{\lambda}>\lambda>1$ two reals, and a cut-off function $\eta$ such that

$$
\eta(x)=\left\{\begin{array}{l}
0 \text { if } x \in \bar{\Omega} \\
1 \text { if } x \in \mathbb{R}^{3} \backslash \lambda \Omega .
\end{array}\right.
$$

Now, since

$$
\Delta(\eta \phi)=2 \nabla \eta \cdot \nabla \phi+\phi \Delta \eta
$$

belongs to $L^{2}\left(\mathbb{R}^{3}\right)$ (notice that $\Delta \eta$ is compactly supported), $\eta \phi$ belongs to $\dot{H}^{2}\left(\mathbb{R}^{3}\right)$.

From this, we derive that the restriction $\left.\phi\right|_{\bar{\lambda} \Gamma}$ of $\phi$ on $\bar{\lambda} \Gamma$ belongs to $H^{3 / 2}(\bar{\lambda} \Gamma)$. To conclude, we notice that $\phi$ verifies

$$
\left\{\begin{array}{l}
\Delta \phi=0 \text { on } \bar{\lambda} \Omega \backslash \Omega, \\
\left.\phi\right|_{\bar{\lambda} \Gamma} \in H^{3 / 2}(\bar{\lambda} \Gamma), \\
\left.\phi\right|_{\Gamma} \in H^{3 / 2}(\Gamma)
\end{array}\right.
$$

and thus $\phi \in H^{2}(\bar{\lambda} \Omega \backslash \bar{\Omega})$. Putting all these results together proves the lemma. 
Lemma 2 (rate of decay). There exist $R>0$ and $C>0$ such that for all $x \geq R$ the Hessian $D^{2} \phi$ of $\phi$ satisfies

$$
\left|D^{2} \phi(x)\right| \leq \frac{C}{|x|^{4}}
$$

Proof. Since $\phi$ is harmonic outside $\Omega$, we can compute its second derivative by differentiating the formula (4).

$$
D^{2} \phi(x)=\frac{1}{4 \pi} \int_{\Omega} \vec{u}(y) \cdot D_{x}^{2}\left(\frac{x-y}{|x-y|^{3}}\right) \mathrm{d} y .
$$

Clearly $D^{2}\left(\frac{x}{|x|^{3}}\right)$ is homogeneous of degree -4 and there exists $C>0$ such that

$$
\left|D^{2}\left(\frac{x}{|x|^{3}}\right)\right|<\frac{C}{|x|^{4}}
$$

Putting this into (13) gives

$$
\left|D^{2} \phi(x)\right| \leq C \int_{\Omega} \frac{|\vec{u}(y)|}{|x-y|^{4}} \mathrm{~d} y .
$$

Now, taking any $R>0$ such that $\bar{\Omega} \subset B(0, R)$ gives the result providing a change of the constant $C$.

We are now in a position to state the theorem that gives the error between $\phi$ and $\phi_{h}$.

Theorem 1. There exists a constant $C$ such that

$$
\left|\phi-\phi_{h}\right|_{1, *} \leq C h_{\mathcal{T} \cup C_{1}}
$$

Proof. We call $N$ the smallest integer such that

$$
B(0, R) \subset \xi^{N} \Omega
$$

with $R$ defined in Lemma 2 .

If we call $\bar{d}=\max _{x \in \partial \Omega}|x|$ and $\underline{d}=\min _{x \in \partial \Omega}|x|$, we have

$$
\xi^{N-1} \underline{d}<R<\xi^{N} \bar{d}
$$

Now, we start from the formula stating the interpolation error, well known for elliptic equations solved by the finite elements method (see [4] for instance)

$$
\left|\phi-\phi_{h}\right|_{1, *}^{2} \leq C \sum_{K \in \overline{\mathcal{T}}}|\phi|_{H^{2}(K)}^{2} h_{K}^{2},
$$

and we make a different estimation according to the fact that the element $K$ is in $\Omega$, or in $C_{n}$ with $n>N$ or $n \leq N$.

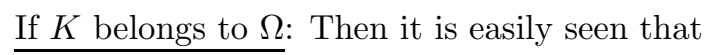

$$
\sum_{K \in \mathcal{T}}|\phi|_{H^{2}(K)}^{2} h_{K}^{2} \leq|\phi|_{H^{2}(\Omega)}^{2} h_{\mathcal{T}}^{2}
$$


$\underline{\text { If } n \leq N}$ : The aspect ratio $h_{K}$ of an element $K$ is bounded by a constant times $h_{C_{1}}$. Indeed $K$ belongs at most to the $N^{t h}$ layer with

$$
\xi^{N} \leq \frac{R \xi}{\underline{d}} \leq C .
$$

The aspect ratio $h_{K}$ of the element $K$ is then bounded by

$$
h_{K} \leq \xi^{N} h_{C_{1}} \leq C h_{C_{1}} .
$$

Summing with respect to $K$ gives

$$
\sum_{K \in C_{1} \cup \cdots \cup C_{N}}|\phi|_{H^{2}(K)}^{2} h_{K}^{2} \leq C|\phi|_{H^{2}\left(C_{1} \cup \cdots \cup C_{N}\right)}^{2} h_{C_{1}}^{2} \leq C|\phi|_{H^{2}(\omega)}^{2} h_{C_{1}}^{2} .
$$

$\underline{\text { If } n>N}$ : Taking into account the elements of the $n$-th layer gives

$$
\sum_{K \in C_{n}}|\phi|_{H^{2}(K)}^{2} h_{K}^{2} \leq|\phi|_{H^{2}\left(C_{n}\right)}^{2} h_{C_{n}}^{2},
$$

and since $n>N$, we have by Lemma 2

$$
|\phi|_{H^{2}\left(C_{n}\right)}^{2} \leq C \int_{C_{n}} \frac{\mathrm{d} x}{|x|^{8}} \leq C \xi^{-8 n} \operatorname{Vol}\left(C_{n}\right) \leq C \xi^{-5 n} \operatorname{Vol}\left(C_{1}\right),
$$

and since

we thus deduce

$$
\operatorname{Vol}\left(C_{1}\right)=\left(\xi^{3}-1\right) \operatorname{Vol}(\Omega)
$$

$$
\sum_{n>N} \sum_{K \in C_{n}}|\phi|_{H^{2}(K)}^{2} h_{K}^{2} \leq C\left(\xi^{3}-1\right) \sum_{n>N} \xi^{-5 n} h_{C_{n}}^{2} \leq C\left(\xi^{3}-1\right) \sum_{n>N} \xi^{-3 n} h_{C_{1}}^{2} \leq \frac{C}{\xi^{N-2}} h_{C_{1}}^{2} \leq C h_{C_{1}}^{2} .
$$

Combining together $(15,16)$ and $(19)$ gives the desired result

$$
\left|\phi-\phi_{h}\right|_{1, *} \leq C h_{\mathcal{T} \cup C_{1}} .
$$

\section{Practical implementation - Truncation of the domain}

From the practical point of view, the method described before cannot be used directly because it needs to solve an infinite dimensional linear system. In order to reduce the problem to a finite dimensional one, we consider the domain $\Omega_{M}=\xi^{M} \Omega$ which consists of the union of $\Omega$ and the first $M$ layers. Such approximations of unbounded problems by bounded ones are currently used (see e.g. $[1,5,8]$ and references therein), and we must provide a condition on the boundary of the outer truncated domain.

The three natural boundary conditions are namely homogeneous Dirichlet, Neumann and mixed boundary conditions. These three boundary conditions are discussed for instance, in [8] (see also the discussion in [1] for the Helmholtz equation) where it is shown that for the Laplace equation with a right hand side which is non compactly supported, the Neumann boundary conditions might give a solution which does not converge as the outer boundary goes to infinity and that the mixed boundary condition is better than the Dirichlet.

We also remark that the mixed boundary condition adapted to our problem (which takes into account the decay of the solution in $\frac{1}{R^{2}}$ instead of the classical $\frac{1}{R}$ decay for such problems) is not as natural to write as the one studied in $[8]$

$$
\frac{\partial \phi}{\partial n}+\frac{(x \cdot n)}{|x|^{2}} \phi=0
$$


Indeed, since

$$
\phi \sim_{x \rightarrow \infty} \frac{1}{4 \pi} \int_{\Omega} \vec{u}(y) \mathrm{d} y \cdot \frac{x}{|x|^{3}}
$$

(see next section), there is no obvious manner to cancel this first term of the expansion with the normal derivative

$$
\frac{\partial \phi}{\partial n} \sim_{x \rightarrow \infty} n(x) \cdot \frac{1}{4 \pi}\left(\frac{I d}{|x|^{3}}-3 \frac{x \otimes x}{|x|^{5}}\right) \int_{\Omega} \vec{u}(y) \mathrm{d} y
$$

in the case $\Omega_{M}$ is not a sphere. In the numerical experiments shown at the end of this paper, the domain is a sphere and thus we can simply write the condition (later on called homogeneous mixed condition)

$$
\frac{\partial \phi}{\partial n}+2 \frac{(x \cdot n)}{|x|^{2}} \phi=0
$$

However, in the general case, several conditions may be written like the non-homogeneous mixed condition

$$
\frac{\partial \phi}{\partial n}+3 \frac{(x \cdot n)}{|x|^{2}} \phi=\frac{1}{4 \pi} \int_{\Omega} \vec{u}(y) \mathrm{d} y \cdot n(x),
$$

but it is not clear what should be the good writing.

The aim of this section is to prove with classical error studies that the Neumann boundary condition on our problem does actually converge (at least in $H^{1}$ seminorm), to explicit the construction of the outer mesh by giving an automatic procedure, and to give a preconditionning technique. The numerical tests are given in the last section.

\subsection{Neumann boundary conditions}

We study from the analytical point of view the approximation of the original problem (2) by the truncated problem

$$
\left\{\begin{array}{l}
\Delta \phi_{M}=\operatorname{div}(\vec{u}) \text { in } \mathcal{D}^{\prime}\left(\Omega_{M}\right) \\
\frac{\partial \phi_{M}}{\partial n}=g \text { on } \partial \Omega_{M}
\end{array}\right.
$$

which is solved by a finite elements procedure applied to the natural mesh $\mathcal{T}_{M}$ of $\Omega_{M}$ consisting of the mesh of $\Omega$ glued to the mesh of the $M$ first layers as described in Section 2. We give an estimate of the error between $\phi_{M}$ and $\phi$ solution to (2), with respect to $M$ the number of layers and $g$ the chosen Neumann boundary condition (notice that in (21) $\phi_{M}$ is defined up to a constant and for having solutions to (21) $g$ must satisfy $\int_{\partial \Omega_{M}} g \mathrm{~d} \sigma=0$ ).

Lemma 3. We call

$$
H_{M}^{1}=\left\{\psi \in H^{1}\left(\Omega_{M}\right) \text { such that } \int_{\Omega_{M}} \psi \mathrm{d} x=0, \text { and }\left.\psi\right|_{K} \in P^{1}(K) \text { for all } K \in \mathcal{T}_{M}\right\} \text {. }
$$

Then the problem

$$
\text { Find } \phi_{M} \in H_{M}^{1} \text {, such that } \int_{\Omega_{M}} \nabla \phi_{M} \cdot \nabla \psi \mathrm{d} x=\int_{\Omega} \vec{u} \cdot \nabla \psi \mathrm{d} x \text { for all } \psi \in H_{M}^{1}
$$

has a unique solution $\phi_{M}$ in $H_{M}^{1}$ which satisfies

$$
\left|\phi-\phi_{M}\right|_{H^{1}\left(\Omega_{M}\right)} \leq C_{1} \inf _{\psi \in H_{M}^{1}}|\phi-\psi|_{H^{1}\left(\Omega_{M}\right)}+C_{2} \xi^{M}\left\|\frac{\partial \phi}{\partial n}-g\right\|_{L^{2}\left(\partial \Omega_{M}\right)},
$$

where $C_{1}$ and $C_{2}$ are independent of $h, M$ and $\xi$. 
Proof. Existence and uniqueness of the solution are classical. In the error estimation, the only point is to explain the last term, and more precisely the dependence of the constant on $\xi$. We call $a_{M}(\phi, \psi)$ the bilinear form defined by

$$
a_{M}\left(\phi_{1}, \phi_{2}\right)=\int_{\Omega_{M}} \nabla \phi_{1} \cdot \nabla \phi_{2} \mathrm{~d} x
$$

and we notice that $\phi$ and $\phi_{M}$ satisfy

$$
a_{M}(\phi, \psi)=\int_{\Omega} \vec{u} \cdot \nabla \psi \mathrm{d} x+\int_{\partial \Omega_{M}} \frac{\partial \phi}{\partial n} \psi \mathrm{d} \sigma, \forall \psi \in H^{1}\left(\Omega_{M}\right)
$$

and

$$
a_{M}\left(\phi_{M}, \psi_{M}\right)=\int_{\Omega} \vec{u} \cdot \nabla \psi_{M} \mathrm{~d} x+\int_{\partial \Omega_{M}} g \psi_{M} \mathrm{~d} \sigma, \forall \psi_{M} \in H_{M}^{1}\left(\Omega_{M}\right) .
$$

These two equations allow us to write

$$
\begin{aligned}
a_{M}\left(\phi-\phi_{M}, \phi_{M}-\psi_{M}\right) & =a_{M}\left(\phi-\psi_{M}+\psi_{M}-\phi_{M}, \phi_{M}-\psi_{M}\right) \\
& =-\int_{\Omega_{M}}\left|\nabla\left(\phi_{M}-\psi_{M}\right)\right|^{2} d x+\int_{\partial \Omega_{M}}\left(\frac{\partial \phi}{\partial n}-g\right)\left(\phi_{M}-\psi_{M}\right) \mathrm{d} \sigma, \quad \forall \psi_{M} \in H_{M}^{1}\left(\Omega_{M}\right),
\end{aligned}
$$

from which we deduce

$$
\begin{aligned}
\int_{\Omega_{M}}\left|\nabla\left(\phi_{M}-\psi_{M}\right)\right|^{2} \mathrm{~d} x \leq & \left\|\nabla\left(\phi-\psi_{M}\right)\right\|_{L^{2}\left(\Omega_{M}\right)}\left\|\nabla\left(\phi_{M}-\psi_{M}\right)\right\|_{L^{2}\left(\Omega_{M}\right)} \\
& +\left\|\frac{\partial \phi}{\partial n}-g\right\|_{L^{2}\left(\partial \Omega_{M}\right)}\left\|\phi_{M}-\psi_{M}\right\|_{L^{2}\left(\partial \Omega_{M}\right)} .
\end{aligned}
$$

Noticing that $\phi_{M}$ and $\psi_{M}$ belong to $H_{M}^{1}$, their trace are in $L^{2}\left(\partial \Omega_{M}\right)$ with

$$
\begin{aligned}
\left\|\phi_{M}-\psi_{M}\right\|_{L^{2}\left(\partial \Omega_{M}\right)} & \leq C_{M}\left\|\phi_{M}-\psi_{M}\right\|_{H^{1}\left(\Omega_{M}\right)} \\
& \leq C_{M}^{\prime}\left|\phi_{M}-\psi_{M}\right|_{H^{1}\left(\Omega_{M}\right)}
\end{aligned}
$$

(this last assertion needs a Poincaré inequality which is valid since $\phi_{M}$ and $\psi_{M}$ satisfy $\int_{\Omega_{M}} \phi_{M}=\int_{\Omega_{M}} \psi_{M}=0$ ).

Making a scaling of this latter inequality (notice that $\Omega_{M}=\xi^{M} \Omega$ ), we get

$$
\| \phi_{M}-\left.\psi_{M}\right|_{L^{2}\left(\partial \Omega_{M}\right)} \leq C \xi^{M}\left|\phi_{M}-\psi_{M}\right|_{H^{1}\left(\Omega_{M}\right)},
$$

where the constant $C$ depends only on the shape of $\partial \Omega$ (and therefore is independant of $M, h$ and $\xi$ ).

Plugging this into (25) gives

$$
\left|\phi_{M}-\psi_{M}\right|_{H^{1}\left(\Omega_{M}\right)} \leq\left|\phi-\psi_{M}\right|_{H^{1}\left(\Omega_{M}\right)}+C \xi^{M}\left\|\frac{\partial \phi}{\partial n}-g\right\|_{L^{2}\left(\partial \Omega_{M}\right)},
$$

from which we get the result using

$$
\left|\phi_{M}-\psi_{M}\right|_{H^{1}\left(\Omega_{M}\right)} \geq\left|\phi_{M}-\phi\right|_{H^{1}\left(\Omega_{M}\right)}-\left|\phi-\psi_{M}\right|_{H^{1}\left(\Omega_{M}\right)} .
$$


The error estimation given above allows us to give an asymptotic number of layers to keep. For instance, if we take homogeneous Neumann boundary conditions, we have the following theorem:

Theorem 2. Using $g=0$, it is sufficient to take a number of layers $M$ varying asymptotically with respect to $h=h_{\mathcal{T} \cup C_{1}}$ as

$$
M \sim_{h \rightarrow 0}-\frac{\ln h}{\ln \xi}
$$

in order to have the same error estimate as before

$$
\left|\phi-\phi_{M}\right|_{H^{1}\left(\Omega_{M}\right)} \leq C h .
$$

Proof. We start from (22) and notice that from the same argument that was used in Section 3, there exists a constant $C$ independant of $M$ and $h$, such that

$$
\inf _{\psi_{M} \in H_{M}^{1}}\left|\phi-\psi_{M}\right|_{H_{M}^{1}} \leq C h
$$

Now, if $M$ is taken sufficiently large to ensure that $\xi^{M} \underline{d}>R$, it is possible to use the asymptotic decay of the derivative of $\phi$ to estimate $\left\|\frac{\partial \phi}{\partial n}\right\|_{L^{2}\left(\partial \Omega_{M}\right)}$. Indeed, from (4), we have

$$
\left|\frac{\partial \phi}{\partial n}\right| \leq \frac{C}{\left(\xi^{M} \underline{d}\right)^{3}} \text { on } \partial \Omega_{M}
$$

which leads to

$$
\left\|\frac{\partial \phi}{\partial n}\right\|_{L^{2}\left(\partial \Omega_{M}\right)} \leq\left(\frac{C \xi^{2 M}}{\xi^{6 M}}\right)^{\frac{1}{2}}
$$

(the term $\xi^{2 M}$ measures the surface of $\partial \Omega_{M}$ with respect to $M$ ).

In order to prove the theorem, we just need to ensure

$$
\frac{\xi^{M}}{\xi^{2 M}} \leq C h
$$

with $C$ independant of $h$.

This precisely gives the asymptotics (27).

Of course, it is possible to improve the preceding result by using an approximation of the normal derivative of $\phi$ for the function $g$. In Section 4.1, we simply took $g \equiv 0$ to get the result. This can be quite bad since $\frac{\partial \phi}{\partial n}$ decays only algebraically. However, since an exact formula is known for $\phi$ (formula (4)), it is possible to get the far-field approximation of $\frac{\partial \phi}{\partial n}$ at infinity. Indeed, since

$$
\phi(x)=\frac{1}{4 \pi} \int_{\Omega} \vec{u}(y) \cdot \frac{x-y}{|x-y|^{2}} \mathrm{~d} y, \quad \forall x \in \omega,
$$


we can compute the first term of the development of $\frac{\partial \phi}{\partial n}$ for large $x$

$$
\begin{aligned}
\frac{\partial \phi}{\partial n}(x) & =n(x) \cdot \nabla \phi(x) \\
& =n(x) \cdot \frac{1}{4 \pi} \int_{\Omega}\left(\frac{I d}{|x-y|^{3}}-3 \frac{(x-y) \otimes(x-y)}{|x-y|^{5}}\right) \vec{u}(y) \mathrm{d} y, \\
& \sim \underset{x \rightarrow \infty}{\sim} n(x) \cdot \frac{1}{4 \pi}\left(\frac{I d}{|x|^{3}}-3 \frac{x \otimes x}{|x|^{5}}\right) \int_{\Omega} \vec{u}(y) \mathrm{d} y+O\left(|x|^{-4}\right),
\end{aligned}
$$

where $I d$ stands for the $3 \times 3$ identity matrix.

Theorem 3. If we take $g(x)=n(x) \cdot\left(\frac{I d}{|x|^{3}}-3 \frac{x \otimes x}{|x|^{5}}\right) \int_{\Omega} \vec{u}(y) d y$, we have

$$
\left\|\frac{\partial \phi}{\partial n}-g\right\|_{L^{2}\left(\partial \Omega_{M}\right)} \leq \frac{C}{\xi^{2 M}}
$$

and thus the asymptotic number of layers required to obtain a good approximation of $\phi$ is half the preceding one

$$
M \sim_{h \rightarrow 0}-\frac{\ln h}{2 \ln \xi} .
$$

Proof. First, it is not difficult to check that

$$
\int_{\partial \Omega_{M}} n(x) \cdot \frac{1}{4 \pi}\left(\frac{I d}{|x|^{3}}-3 \frac{x \otimes x}{|x|^{5}}\right)=0
$$

so that the proposed boundary condition is valid. Indeed, for the $i$-th component

$$
\int_{\partial \Omega_{M}} \frac{1}{4 \pi}\left(\frac{n_{i}(x)}{|x|^{3}}-3 \frac{x_{i}(x \cdot n)}{|x|^{5}}\right)=\int_{\partial \Omega_{M}} \frac{\partial}{\partial x_{i}}\left(\frac{x}{|x|^{3}}\right) \cdot n(x) \mathrm{d} x=\int_{\Omega_{M}} \frac{\partial}{\partial x_{i}} \operatorname{div}\left(\frac{x}{|x|^{3}}\right) \mathrm{d} x=0
$$

since $\frac{x}{|x|^{3}}$ is divergence free.

Then, with the Taylor expansion of $\frac{\partial \phi}{\partial n}$ at infinity given by (29), we deduce

$$
\left\|\frac{\partial \phi}{\partial n}-g\right\|_{L^{2}\left(\partial \Omega_{M}\right)} \leq C\left(\frac{\xi^{2 M}}{\xi^{8 M}}\right)^{\frac{1}{2}}=\frac{C}{\xi^{3 M}},
$$

and the asymptotics (30) is simply done as before in order to ensure that

$$
\frac{\xi^{M}}{\xi^{3 M}} \leq C h
$$

with $C$ independent of $h$.

\subsection{Impact on Implementation}

Frome the practical point of view, one of the main advantages of the method presented here is its low cost storage. Indeed, it has been already mentionned by Ying [12] that the rigidity matrix of the outer mesh does not need to be fully stored and can be reconstructed from the knowledge of the matrix of the first layer only (which is even sparse). 
If we call $\phi$ a $P^{1}$ function on the graded mesh, whose unknowns are denoted by $\phi_{0}$ on $\Gamma, \phi_{1}$ on $\xi \Gamma$, and more generally $\phi_{i}$ on $\xi^{i} \Gamma$, and denoting by $R=\left(\begin{array}{cc}K & A^{T} \\ A & K^{\prime}\end{array}\right)$ the rigidity matrix on $C_{1}$, we get the following expression

$$
\int_{C_{1}}|\nabla \phi|^{2} \mathrm{~d} x=\left(\begin{array}{ll}
\phi_{0}^{T} & \phi_{1}^{T}
\end{array}\right)\left(\begin{array}{cc}
K & A^{T} \\
A & K^{\prime}
\end{array}\right)\left(\begin{array}{c}
\phi_{0} \\
\phi_{1}
\end{array}\right)
$$

Now, since all the layers are homothetic to $C_{1}$, we get

$$
\int_{C_{i}}|\nabla \phi|^{2} \mathrm{~d} x=\left(\phi_{i-1}^{T} \phi_{i}^{T}\right) \xi^{i-1}\left(\begin{array}{cc}
K & A^{T} \\
A & K^{\prime}
\end{array}\right)\left(\begin{array}{c}
\phi_{i-1} \\
\phi_{i}
\end{array}\right)
$$

and therefore the total rigidity matrix can be reassembled from the knowledge of $R$ only. For instance the energy on $C_{1} \cup \cdots \cup C_{M}$ is equal to

$$
\int_{C_{1} \cup \ldots \cup C_{M}}|\nabla \phi|^{2} d x=\sum_{i=1}^{M}\left(\phi_{i-1}^{T} \phi_{i}^{T}\right) \xi^{i-1}\left(\begin{array}{cc}
K & A^{T} \\
A & K^{\prime}
\end{array}\right)\left(\begin{array}{c}
\phi_{i-1} \\
\phi_{i}
\end{array}\right),
$$

which gives a matrix for the outer part

$$
\mathcal{A}=\left(\begin{array}{ccccc}
K & A^{T} & 0 & \cdots & 0 \\
A & K^{\prime}+\xi K & \xi A^{T} & \ddots & 0 \\
0 & \xi A & \xi K^{\prime}+\xi^{2} K & \ddots & 0 \\
\vdots & \vdots & \ddots & \ddots & \vdots \\
0 & \cdots & \cdots & \xi^{M} A & \xi^{M} K^{\prime}
\end{array}\right)
$$

From the implementation point of view, the main drawback comes from the multiplication by this power of $\xi$. Moreover, since $\xi>1$, for very big values of $M$, the matrix $\mathcal{A}$ is not well balanced, and a disproportion in the coefficients between first lines and last lines may lead to numerical instabilities.

For these reasons, we simply rewrite the energy making the change of variables

$$
\psi_{i}=\phi_{i} \xi^{\frac{i}{2}}
$$

We have

$$
\begin{aligned}
\int_{C_{1} \cup \cdots \cup C_{M}}|\nabla \phi|^{2} \mathrm{~d} x & =\sum_{i=1}^{M}\left(\phi_{i-1}^{T} \phi_{i}^{T}\right) \xi^{i-1}\left(\begin{array}{cc}
K & A^{T} \\
A & K^{\prime}
\end{array}\right)\left(\begin{array}{c}
\phi_{i-1} \\
\phi_{i}
\end{array}\right), \\
& =\sum_{i=1}^{M}\left(\psi_{i-1}^{T} \psi_{i}^{T}\right)\left(\begin{array}{cc}
\bar{K} & \bar{A}^{T} \\
\bar{A} & \bar{K}^{\prime}
\end{array}\right)\left(\begin{array}{c}
\psi_{i-1} \\
\psi_{i}
\end{array}\right),
\end{aligned}
$$

where $\bar{K}=K, \bar{A}=\frac{A}{\sqrt{\xi}}$, and $\bar{K}^{\prime}=\frac{K^{\prime}}{\xi}$. 
This gives a matrix in the linear system which is almost block-Toeplitz

$$
\mathcal{B}=\left(\begin{array}{ccccc}
\bar{K} & \bar{A}^{T} & 0 & \cdots & 0 \\
\bar{A} & \bar{K}^{\prime}+\bar{K} & \bar{A}^{T} & \ddots & 0 \\
0 & \bar{A} & \bar{K}^{\prime}+\bar{K} & \ddots & 0 \\
\vdots & \vdots & \ddots & \ddots & \vdots \\
0 & \cdots & \cdots & \bar{A} & \overline{\bar{K}}^{\prime}
\end{array}\right)
$$

and seems better balanced.

We will see in the numerical study below that this change of variables not only simplifies the implementation, but acts like a preconditionner. The number of iterations needed to solve the linear system coming from the discretization will be reduced by a significant factor (of course in order to take into account the boundary condition at infinity, we have to express the chosen boundary condition in terms of the $\psi_{i}^{\prime} s$. This changes a little bit the matrix and has not been presented here).

\subsection{Automatic construction of the exterior mesh}

Since we only need to compute the rigidity matrix on the first layer, we describe a simple algorithm to build automatically this layer. We start from the knowledge of a mesh $\mathcal{T}_{\mathcal{B}}$ of the boundary $\Gamma$ which consists of triangles. We call the nodes of $\mathcal{T}_{\mathcal{B}}, S_{1}$ to $S_{N_{B}}$. The nodes of the boundary of the first layer $C_{0}$ are thus given half by $S_{1}$ to $S_{N_{B}}$, and half by $\xi S_{1}$ to $\xi S_{N_{B}}$ (where $\xi S$ means the point of coordinates $\left(\xi x_{S}, \xi y_{S}, \xi z_{S}\right)$, if $\left(x_{S}, y_{S}, z_{S}\right)$ are the coordinates of $S$ and the origin $O$ is the center of the homothetic transformation). Linking $S_{i}$ to $\xi S_{i}$ gives a natural mesh of $C_{0}$ made by prisms. Now, the problem is that we need to cut these prisms into tetraedra in a consistent way (this means that two neighbouring prisms must share a face which is cut in the same way from each side, see Fig. 2). We do that in the following way.

For each prism with vertices $S_{i}, S_{j}, S_{k}, \xi S_{i}, \xi S_{j}$ and $\xi S_{k}$, where we may assume $i<j<k$, we build the three tetraedra with vertices $\left(S_{i}, S_{j}, S_{k}, \xi S_{k}\right),\left(S_{i}, S_{j}, \xi S_{j}, \xi S_{k}\right)$, and $\left(S_{i}, \xi S_{i}, \xi S_{j}, \xi S_{k}\right)$. This is now clear that on the face delimited by $S_{j}, S_{k}, \xi S_{j}$ and $\xi S_{k}$ we created an edge $\left(S_{j}, \xi S_{k}\right)$. Since $j<k$ also for the neighbouring tetraedron, the algorithm given above will make exactly the same cut in the face $\left(S_{j}, S_{k}, \xi S_{j}, \xi S_{k}\right)$ when considered from the point of view of the second prism (see Fig. 2 for a complete picture).

\section{Numerical RESUlts}

The algorithm described before is checked on the well-known situation of the spherical unit ball uniformly magnetized. This case is not very severe, but we just give it in order to illustrate the methodology. Let us consider $\Omega=B(0,1)$ and $\vec{u}=(0,0,1)$ is a constant vectorfield. The exact demagnetizing field can be computed [7] and is given by

$$
H=\nabla \phi=\frac{1}{3} \vec{u},
$$

inside $\Omega$ and decays outside to 0 (the formula outside $\Omega$ can also be computed [7]).

In Figure 5, we have drawn the trace on $\partial \Omega$ of a typical mesh of the ball used for the computation. The mesh is the second mesh whose characteristics are given in Table 1.

Moreover the result in terms of $\phi$ is also given in Figure 5. We have plotted the isosurface of the solution in the ball. Since the solution has a gradient which is a constant vertical vectorfield, the isosurface are simply parallel horizontal discs.

For the study below, three meshes of the unit ball have been used. The characteristics of all the meshes are given in Table 1 in terms of number of vertices, number of tetraedra, number of vertices on the boundary and 


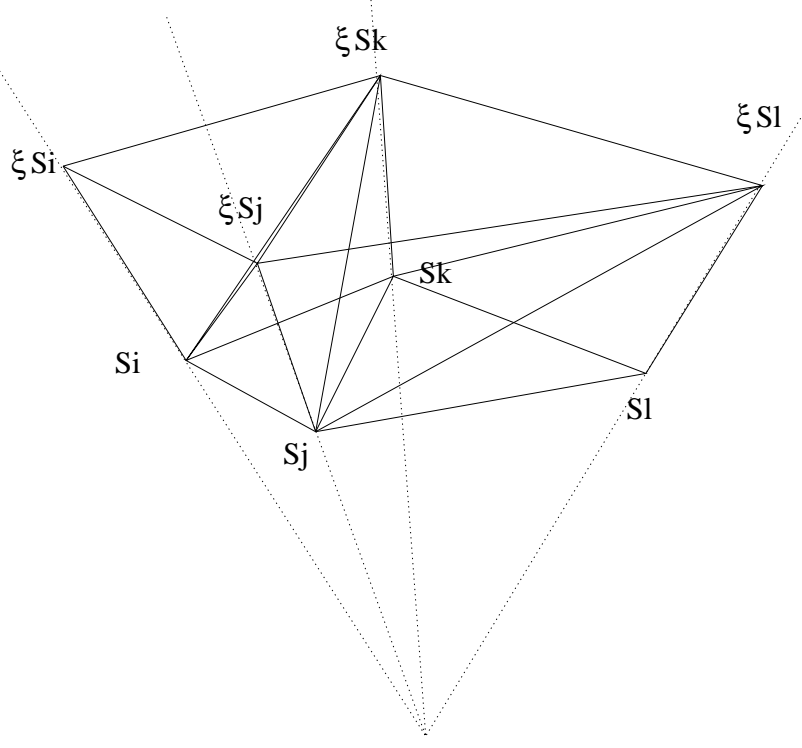

FIGURE 2. Cutting two neighbouring prisms into tetraedra. We have assumed in this picture $i<j<k<l$.
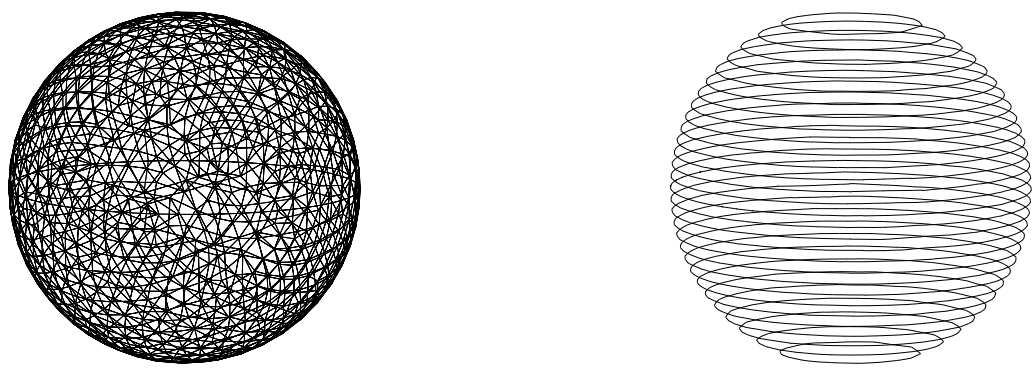

Figure 3. Mesh of the ball and isovalue curves for $\phi$.

TABLE 1. Characteristics of the three meshes that have been used.

\begin{tabular}{|c|c|c|c|c|}
\hline Mesh & vertices & tetraedra & vertices on bdry & mesh size \\
\hline Mesh1 & 1383 & 6899 & 440 & 0.17 \\
\hline Mesh2 & 3458 & 17627 & 1030 & 0.11 \\
\hline Mesh3 & 24361 & 134053 & 4257 & 0.06 \\
\hline
\end{tabular}

mesh size of the mesh. We see that the meshes are finer and finer. Moreover, for all the computations done in this section, the homothetic factor has been always taken equal to $\xi=1.1$.

In a first test, we compare the number of iterations needed to solve the linear system coming from the discretization whether or not we use the preconditionning technique described above.

For this, we use the second mesh of the unit ball, build the outer mesh with an increasing number of layers and compare in terms of number of iterations needed to solve the system the preconditionned method and the non-preconditionned one. In order to do the comparison, we supply in both cases the same mixed boundary conditions, and no other precontionning technique has been added. We further precise that the linear system 
is solved by using the conjugate gradients method. The graph shown Figure 4 shows the number of iterations needed in the conjugate gradients method to reduce the norm of the residual by a factor of $10^{-6}$ using both methods with respect to the number of layers kept in the discretization.

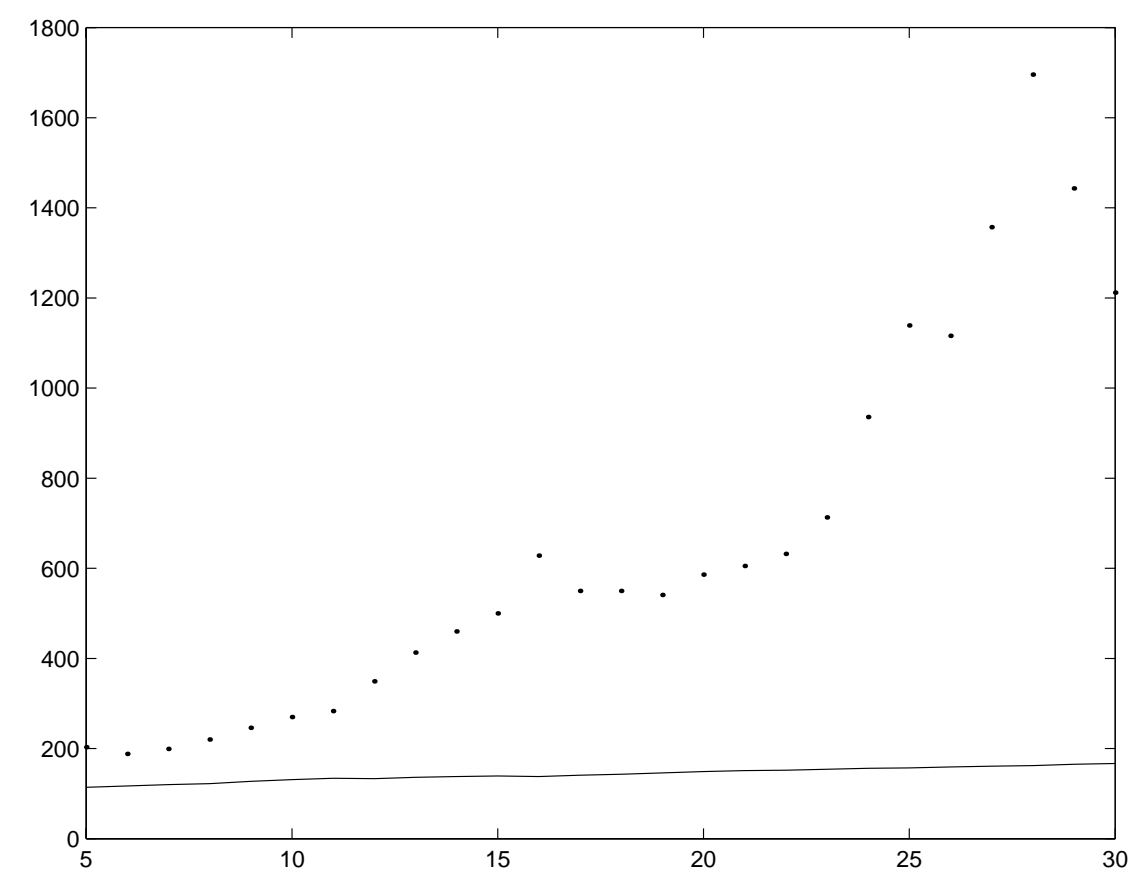

FiguRE 4. Number of iterations for the non-preconditionned (.) and preconditionned (-) conjugate gradients with respect to the number of layers.

The aim of the following study is to compare the three boundary conditions in terms of precision and convergence. In order to measure this, we solve the problem using the preconditionned procedure described above and the three possible boundary conditions on the outer mesh: homogeneous Neumann, homogeneous Dirichlet and homogeneous mixed. The Figures 5, 6 and 7 plot for the three meshes described before the error

$$
E r r=\int_{B(0,1)}\left|\nabla \phi-\left(\begin{array}{c}
0 \\
0 \\
\frac{1}{3}
\end{array}\right)\right|^{2} \mathrm{~d} x
$$

in logarithmic scale versus the number of layers kept in the formulation.

We see that with mixed boundary conditions the convergence is already achieved with a very small number of layers whereas the convergence is slower for Dirichlet boundary conditions and slightly yet slower for Neumann. Surprisingly, although Neumann boundary condition needs a relatively big number of layers to achieve a result independent of additional layers, there is always a "bump" in the graphs, showing that the solution obtained for a smaller number of layers may be better than both mixed and Dirichlet boundary conditions.

The influence of the mesh size is obtained by comparing the three plots. We see that the finer the mesh, the bigger the number of layers is needed. However, this number increases very slightly as the mesh size decreases. This confirms at least formally the logarithmic dependance found in the previous section (see also [6]). 


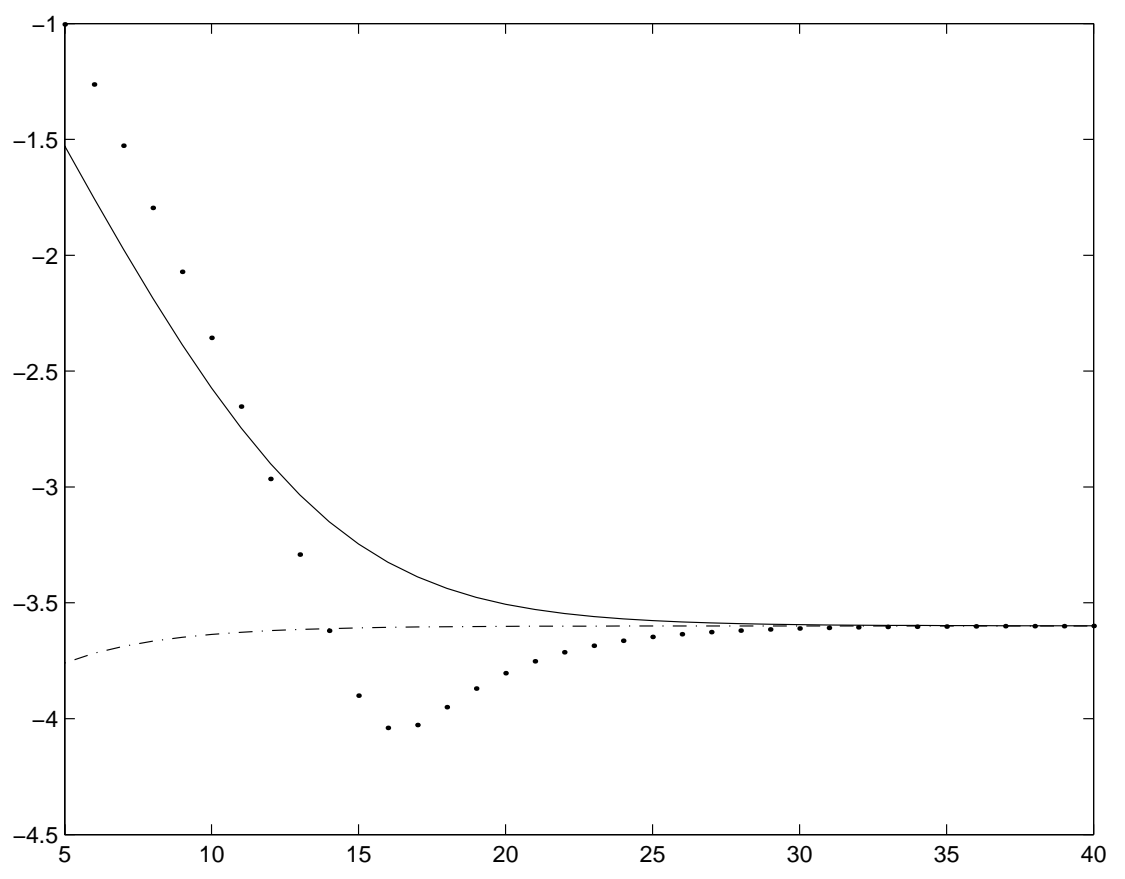

FiguRE 5. Logarithm of Err for the first mesh w.r.t. the number of layers. Neumann (.), Dirichlet (-) and mixed (-.) boundary conditions.

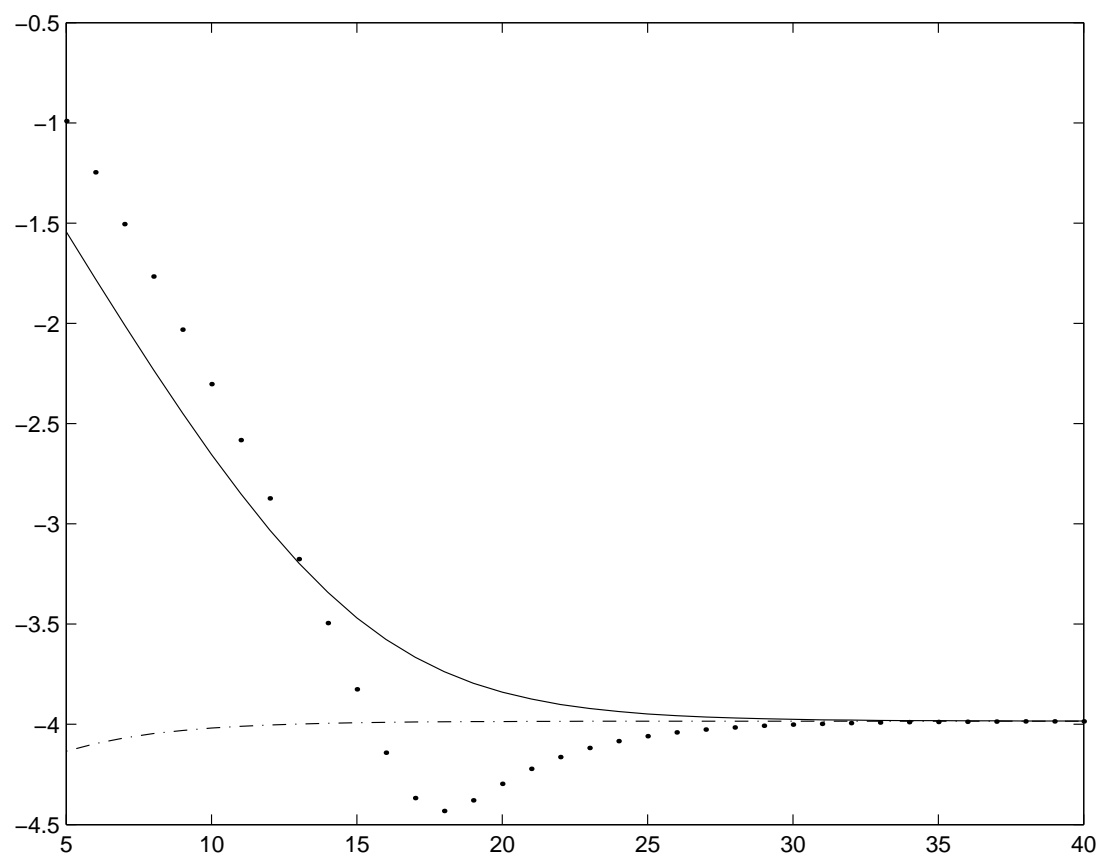

FiguRE 6. Logarithm of Err for the second mesh w.r.t. the number of layers. Neumann (.), Dirichlet (-) and mixed (-.) boundary conditions. 


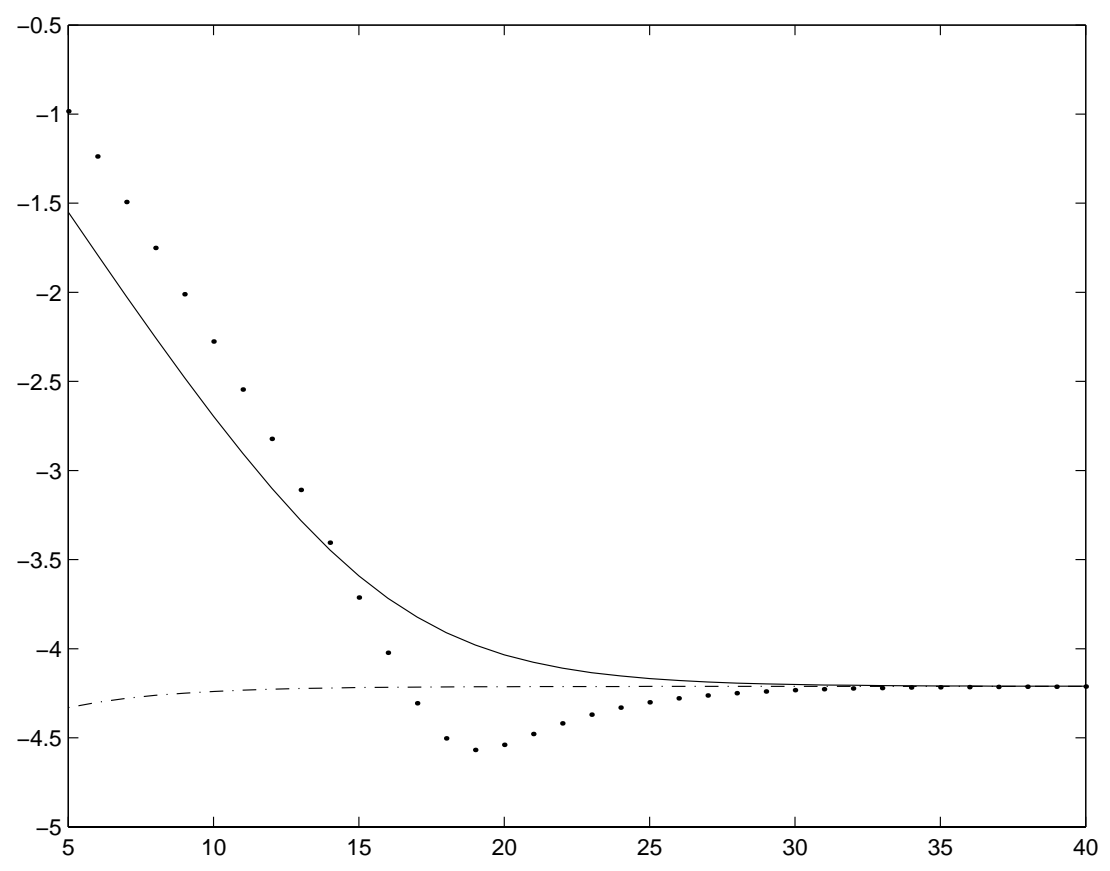

FiguRE 7. Logarithm of Err for the third mesh w.r.t. the number of layers. Neumann (.), Dirichlet (-) and mixed (-.) boundary conditions.

\section{Conclusion}

We have presented a finite element method for the computation of the demagnetizing potential of a ferromagnetic body which is a coupling of a finite elements (for the interior problem), infinite elements for the exterior problem and suitable truncation (and boundary conditions applied on the artificial boundary) of the domain. The advantages of the method are that it is fully automatic and requires a very small storage (the outer region is taken into account with very few unknowns and the matrix is very small). Moreover, we give a very simple preconditionning technique which turns out to make the method really usable. Thus, the method can be applied to quite fine meshes on very low cost computers and for physically relevent 3D computations and gives a natural alternative to boundary elements methods. The main drawback is that it seems up to now restricted to convex or at least star-shaped bodies.

The author would like to thank Jean-Paul Dumeau from the Laboratoire de Mécanique et de Technologie (Cachan, France) as well as Pascal Frey (INRIA) for the meshes of the balls. He also addresses special thanks to Jacques Miltat and André Thiaville from the Laboratoire de Physique des Solides (Orsay, France) who gave him his interest to this subject.

\section{REFERENCES}

[1] A. Bayliss, M. Gunzburger and E. Turkel, Boundary conditions for the numerical solution of elliptic equations in exterior domains. SIAM J. Appl. Math. 42 (1982).

[2] W.F. Brown, Micromagnetics. Interscience Publishers, Wiley \& Sons, New-York (1963).

[3] X. Brunotte, G. Meunier and J.-F. Imhoff, Finite element modeling of unbounded problems using transformations: A rigorous, powerful and easy solution. IEEE Trans. Mag. 28 (1992).

[4] R. Dautray and J.-L. Lions, Analyse mathématique et calcul numérique pour les sciences et les techniques, Vol. 6. Masson, Paris (1988). 
[5] D. Givoli, Non-reflecting boundary conditions. J. Comp. Phys. 94 (1991) 1-29.

[6] C.I. Goldstein, The finite element method with nonuniform mesh sizes for unbounded domains. J. Math. Comput. 36 (1981) 387-404.

[7] J.D. Jackson, Classical Electrodynamics. Wiley and Sons, $2^{\text {nd }}$ edition (1975).

[8] S.A. Nazarov and M. Specovius-Neugebauer, Approximation of exterior problems. Optimal conditions for the Laplacian. Analysis 16 (1996) 305-324.

[9] T. Shreff, Numerische Simulation von Ummagnetisierungsvorgängen in hartmagnetischen Materialen, Ph.D. Thesis. Technische Universität Wien (1993).

[10] R. Fisher, T. Shreffl, H. Kronmüller and J. Fidler, Phase distribution and computed magnetic properties of high-remanent composite magnets. J. Magnetism and Magnetic Materials 150 (1995) 329-344.

[11] P.P. Silvester, D.A. Lowther, C.J. Carpenter and E.A. Wyatt, Exterior finite elements for 2-dimensional field problems with open boundaries. Proc. IEE 124 (1977).

[12] L.A. Ying, Infinite Elements Method. Peking University Press. 